\title{
Open Access, Open Content und gemeinfreies Kulturerbe
}

\author{
Einleitung
}

Seit Beginn der 2000er Jahre haben Open Access und Open Content in der global vernetzten Informationswelt sprunghaft an Bedeutung gewonnen. Die Verfügbarkeit von Wissen ist zu einem entscheidenden Entwicklungsfaktor für Wissenschaft, Wirtschaft, Kultur und Gesellschaft geworden. Angesichts der wachsenden Bedeutung stellen sich Fragen der Verfügbarkeit sowie der Teilhabe auf regionaler, nationaler und globaler Ebene. Der freie Zugang zu Wissen erhöht nachweislich dessen Sichtbarkeit. ${ }^{1}$ Er ist Voraussetzung für technische Erschließungsverfahren und eröffnet neue Möglichkeiten der Auffindbarkeit und automatisierten Verknüpfung.

Aus wissenspolitischer Perspektive trägt Open Access ${ }^{2}$ dazu bei, die drohende Kluft zwischen öffentlich geförderten Informationseinrichtungen in regionalen und nationalen Informationsverbünden zu verringern, zudem ermöglicht Open Access strukturell unterentwickelten Regionen, am wissenschaftlichen und wirtschaftlichen Fortschritt teilzuhaben. Formelle Unterstützung findet Open Access durch führende Wissenschafts- und Fördereinrichtungen wie beispielsweise die Deutsche Forschungsgemeinschaft, die Europäische Kommission sowie den Weltverband der Bibliotheken IFLA.

Im wissenschaftlichen Bereich wird Open Access in Forschungseinrichtungen in den USA, Großbritannien und der Schweiz durch sog. Mandatories (i. e. rechtsverbindliche Verpflichtungen) getragen, die von ihren Wissenschaftlern die Publikation unter Open-Access-Bedingungen einfordern. Hierbei gibt es jedoch erhebliche nationale Unterschiede, die auf verschiedenen Rechtssystemen und Publikationstraditionen gründen.

Der Fokus dieses Beitrags ist darauf gerichtet, wie Wissenschaftliche und Öffentliche Bibliotheken Open Access, Open Content und gemeinfreies Kulturerbe erwerben, bereitstellen, in ihre vorhandenen digitalen Systeme einbinden und unterstützen können. Die Möglichkeiten zukünftiger Tätigkeitsfelder sind hier vielfältig, die Diskussion darüber steht gerade in Deutschland erst am Anfang.

\section{Open Access, Open Content und gemeinfreies Kulturerbe - Definition und Abgrenzung}

Open Access bezeichnet den freien Zugriff zu wissenschaftlichen Publikationen über das Internet sowie deren unbeschränkte Nachnutzbarkeit. Auch wenn der Begriff oft ungenau verwendet wird, gilt die Definition der Berliner Erklärung von 2003 heute als weitgehend maßgeblich. Wir zitieren aus der deutschen Übersetzung von 2006:

\footnotetext{
Open Access-Veröffentlichungen umfassen originäre wissenschaftliche Forschungsergebnisse ebenso wie Ursprungsdaten, Metadaten, Quellenmaterial, digitale Darstellungen von Bild- und Graphik-Material und wissenschaftliches Material in multimedialer Form. [...] Die Urheber und die Rechteinhaber solcher Veröffentlichungen gewähren allen Nutzern unwiderruflich das freie, weltweite Zugangsrecht zu diesen Veröffentlichungen und erlauben ihnen, diese Veröffentlichungen - in jedem beliebigen digitalen Medium und für jeden verantwortbaren Zweck - zu kopieren, zu nutzen, zu verbreiten, zu übertragen und öffentlich wiederzugeben sowie Bearbeitungen davon zu erstellen und zu verbreiten, sofern die Urheberschaft korrekt angegeben wird. ${ }^{3}$
}

1 Vgl. Brody u. Harnad 2004; Brody, Harnad u. Carr 2005; Eysenbach 2006

2 In Einleitung und Ausblick dieses Beitrags verwenden wir den Begriff „Open Access“ im (weiteren) Sinne von offenem Zugang zu Information, Wissenschaft und Kultur.

3 Berliner Erklärung über den offenen Zugang zu Wissen 2003. 
Wenn von Open Access die Rede ist, geht es meistens um neue (,born digital $\left.{ }^{\circ}\right)$ Literatur und um die neuen Möglichkeiten und Herausforderungen im Zusammenhang mit Geschäftsmodellen, Qualitätssicherung und Verbreitung von wissenschaftlicher Information.

Unter Open Content (auch: freie Inhalte) werden frei zugängliche Born-digital-Ressourcen verstanden. Der Begriff ist weiter als Open Access gefasst, da er nicht nur wissenschaftliche Informationen adressiert. Insbesondere partizipative Webanwendungen wie die Wikipedia - die explizit keine originären Forschungsergebnisse aufnimmt - haben zu einer Fülle von Veröffentlichungen aller Art geführt, die vom Entstehungszeitpunkt an explizit frei zugänglich sind.

Die Urheber erreichen dies z. B., indem sie Creative-Commons-Lizenzen (CC-Lizenz) auf ihre Werke anwenden. Solche Lizenzen sollen die Nachnutzbarkeit von Publikationen rechtssicher und klar verständlich regeln. Allerdings soll Creative Commons, anders als Open Access, gemäß der Berliner Erklärung dem Urheber freistellen, z. B. die Weitergabe seines Werks in veränderter Form oder die kommerzielle Nachnutzung zu untersagen.

Ein Sonderfall des Open Content sind die Open Educational Resources (OER). Dieser 2002 von der UNESCO geprägte Begriff bezeichnet Bildungsmaterialien - darunter auch Software - die durch freie Lizenzen und offene Formate möglichst flexibel in verschiedenen Lehr- und Lernsituationen eingesetzt werden können. Das Konzept adressiert vor allem die Entwicklungsländer. Eng verwandt ist das ebenfalls 2002 begründete MIT OpenCourseWare, das mittlerweile zu einem fächerübergreifenden Konsortium von hunderten Hochschulen weltweit angewachsen ist, die digitale Lehrbücher, Lerneinheiten, Vorlesungsmitschnitte etc. unter freien Lizenzen zur Verfügung stellen. ${ }^{4}$

Was wird unter gemeinfreiem Kulturerbe verstanden? Das Urheberrecht in Deutschland sieht vor, dass ein Werk 70 Jahre nach dem Tod seines Urhebers gemeinfrei wird (§ 64 UrhG). Es fällt damit quasi in den Allgemeinbesitz. Jeder kann das Werk dann beliebig benutzen, verändern und - auch kommerziell - weiterverbreiten. Die urheberrechtliche Situation in anderen Ländern ist grundsätzlich ähnlich. $\mathrm{Ob}$ z. B. eine Fotografie eines alten Meisters, die Ende des 20. Jahrhunderts erstellt wurde, ebenfalls gemeinfrei ist, bleibt in der Auslegung des urheberrechtlichen Schutzes umstritten.

Die (Retro-)Digitalisierung dieses gemeinfreien kulturellen Erbes ist ein wichtiger Schritt, um den Zugang zu überlieferten Kulturgütern sowohl für die Forschung als auch für die interessierte Allgemeinheit via Internet langfristig zu sichern und zugleich die Originale durch diese parallele Nutzungsform besser zu schützen. Nutzen und Schaden verschiedener Geschäftsmodelle der (Massen-)Digitalisierung werden heute intensiv diskutiert.

Neben dem Kulturerbe sind mittlerweile in vielen Ländern Amtsschriften, aber auch sämtliche sonstige Daten, die von Ämtern und Behörden produziert werden, durch Informationsfreiheitsgesetze als gemeinfrei definiert. Sie sollen systematisch durch das Internet frei zugänglich gemacht werden. In diesem Zusammenhang ist oft von Open Government (Data) die Rede.

\section{Open Access}

\subsection{Die Situation im deutschen Urheberrecht}

Abgesehen von der Gemeinfreiheit der Werke 70 Jahre nach dem Tod des Urhebers ( $\$ 64$ UrhG) bleibt die Verbreitung und öffentliche Zugänglichmachung wissenschaftlicher Publikationen im deutschen Urheberrecht auch im Open Access zunächst an die Position des Urhebers gebunden. ${ }^{5}$ Dieser darf, genauso wie er durch die Wahl der Publikationsform den Zugang verbieten kann, diesen Zugang durch Lizenzvereinbarungen erlauben. Rechtlich gesehen stellt Open Access somit den Übergang vom Verbot oder der Gestattung

4 http://ocw.mit.edu/index.htm (2.1.2012).

5 Vgl. Steinhauer 2010. 
Open Access, Open Content und gemeinfreies Kulturerbe

nur gegen Bezahlung zur Gestattung ohne Lizenzgebühren dar. Der Inhalt bleibt frei, auch wenn er von dem Erstnutzer kommerziell weiterlizenziert wird. Diese Produzenten-Orientierung des deutschen Urheberrechts, die auf einer Trennung in Produzenten und Nutzer basiert und die sich durch eine zukünftig stärkere Beteiligung der Nutzer an der Wissensproduktion relativieren wird, wurde zuletzt von verschiedenen Seiten grundlegend in Frage gestellt. ${ }^{6}$

Der Urheber hat gemäß deutschem Urheberrecht die Freiheit, seine Werke in der von ihm bevorzugten Form zu veröffentlichen. ${ }^{7}$ Somit bleiben für die Förderung von Open Access die Autoren entscheidend, die für die Idee des Open Access gewonnen werden müssen. Aus diesem Grund kann Open Access in Deutschland bisher auch nicht durch Mandatories durchgesetzt werden, in denen die Urheber von den sie finanzierenden Institutionen zur Veröffentlichung unter Open-Access-Bedingungen verpflichtet werden. Möglich wäre hier allenfalls eine rechtliche Neuordnung im Sinne eines unabdingbaren Zweitveröffentlichungsrechts in der anstehenden Novellierung des Urheberrechts („Dritter Korb“). Darüber hinaus besteht für Forschungseinrichtungen die Möglichkeit, eine Anbietungspflicht an die Institution durchzusetzen (etwa an der Berlin-Brandenburgischen Akademie der Wissenschaften) oder die Publikation unter Open-AccessLizenzen zur Förderauflage zu machen (realisiert beispielsweise durch die US-amerikanischen National Institutes of Health $-\mathrm{NIH}^{8}$ ).

Diese Auflagen widersprechen jedoch den Prinzipien des deutschen Urheberrechts in seiner jetzigen Form. So beschränkt sich auch die Deutsche Forschungsgemeinschaft (DFG) als wichtigste Förderorganisation der Wissenschaft trotz der frühen Unterzeichnung der Berliner Erklärung auf die Form einer deutlich formulierten Erwartung gegenüber den Förderberechtigten.

\subsection{Wege und Geschäftsmodelle}

Der entgeltfreie Zugang zu wissenschaftlicher Information zieht zwangsläufig eine Umverteilung der bei der Publikation anfallenden Kosten nach sich. Prinzipiell wird dabei zwischen dem Goldenen und dem Grünen Weg unterschieden:

- $\quad$ zwischen direkten Open-Access-Veröffentlichungen etwa in einem Open Access-Verlag, einem Open Access-Journal oder auf einer geeigneten Publikationsplattform (Goldener Weg) und

- der Zweitveröffentlichung und Archivierung als Pre-, Parallel- oder Postprint auf einem disziplinären oder institutionellen Repository (Grüner Weg), das in der Regel von einer öffentlich finanzierten Einrichtung (Forschungseinrichtung, Wissenschaftliche Bibliothek) betrieben wird.

Während der Grüne Weg in den letzten Jahren massiv an Bedeutung zugenommen hat und in einigen Fachdisziplinen (z. B. der Physik und den Wirtschaftswissenschaften) zu einem etablierten Schritt in der Publikationskette geworden ist, sind die Publikationsmöglichkeiten im Goldenen Weg noch im Aufbau begriffen. Allerdings gibt es bei der Finanzierung heute eine Vielzahl unterschiedlicher Modelle, die eine OpenAccess-Veröffentlichung auf dem Goldenen Weg ermöglichen. Diese umfassen die Autorenfinanzierung zu Beginn des Publikationsprozesses (Author-Pays-Modell) sowie die Finanzierung durch Förderorganisationen, Fachgesellschaften, institutionelle Mitgliedschaften und Publikationsfonds, die von Universitäten und Forschungseinrichtungen aufgebaut werden. Zudem gehen zahlreiche Institutionen dazu über, ihren Wissenschaftlern eigene Publikationsplattformen (etwa im Rahmen von Universitätsverlagen) zur Verfügung zu stellen. Möglich ist Open Access mittlerweile auch als Teil einer kommerziellen Verwertungskette (vgl. etwa die Publikationen von Hamburg University Press auf der Plattform PaperC ${ }^{9}$ ).

Einen Überblick über die Publikationsmodelle, die vielfach auch im Sinne eines hybriden Publizierens kombiniert werden, bietet die Informationsplattform Open Access. ${ }^{10}$ Festzuhalten bleibt, dass Open Access

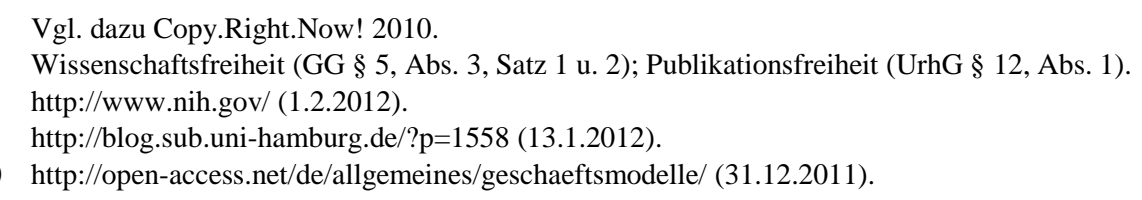


Dienste den Untersuchungen des Public Knowledge Projekts ${ }^{11}$ zufolge durch internettypische Skaleneffekte sowie die Verwendung freier Software Kosteneinsparungen im Vergleich zu gängigen Subskriptionsmodellen erwarten lassen. ${ }^{12}$

\section{Informationspools und Aggregatoren}

Open Access, Open Content und gemeinfreies Kulturerbe - all dies steht im Netz frei zur Verfügung. Doch wie, wo und durch wen werden diese Inhalte jeweils gesammelt und erschlossen?

\subsection{Open Content}

Das bekannteste frei zugängliche Born-digital-Werk im Netz ist die Wikipedia. ${ }^{13}$ Dieses seit 2001 von vielen Internet-Nutzern gemeinschaftlich geschriebene, nichtkommerzielle Lexikon verwendete zunächst die GNU Free Documentation Licence (GFDL) ${ }^{14}$ Seit 2009 steht es zusätzlich unter der Lizenz CC-BYSA. ${ }^{15}$

Die Wikipedia wird in einem beständig fortgeführten Prozess erweitert und umgeschrieben. Die WikiTechnik gewährleistet, dass dennoch auf jeden Lexikonartikel in jedem Stadium persistent verwiesen werden kann. Die starke Vernetzung der Artikel untereinander sowie durch das Kategoriensystem hat Konsequenzen für die Sichtbarkeit der Artikel im Netz. Für viele Stichwortkombinationen, nach denen mit populären Websuchmaschinen gesucht wird, gehört der einschlägige Wikipedia-Artikel zu den ersten Treffern. Daher ist die Wikipedia de facto ein prominenter Rechercheeinstieg. Bibliographien und Linklisten zu den Artikeln sowie die thesaurusartige Struktur der Wikipedia selbst unterstützen die Benutzung von Wikipedia-Artikeln als Ausgangspunkt zur Nutzung weiterer Informationsressourcen.

Diesen Effekt greift das SeeAlso-Plugin auf, mit dem heute von vielen Bibliotheks-(Verbund)Katalogen dynamisch auf die Wikipedia verlinkt wird. ${ }^{16}$ Wenn anhand der ISBN eines Buchs dessen Erwähnung in einem Wikipedia-Artikel ermittelt werden kann, wird der Link auf den bzw. diese Artikel in die Titelvollansicht des jeweiligen Buchs im Katalog eingebunden. Die Einbindung eines an sich dynamischen, unabgeschlossenen Systems wie die Wikipedia in bibliothekarische Entdeckungs-Umgebungen ist auf diese Weise gut gelöst.

Andere Initiativen wie das Personennamendatei-Projekt von Wikipedia und Deutscher Nationalbibliothek $^{17}$ sowie die Freigabe von 250000 Bild-Digitalisaten der Deutschen Fotothek auf der WikipediaPlattform ${ }^{18}$ verfolgen quasi das umgekehrte Ziel, bibliothekarisch erschlossene Wissensräume von der Wikipedia aus besser sichtbar und einfacher zugänglich zu machen. Ideen für weitere Projekte dieser Art ${ }^{19}$ weisen auf das hohe Potenzial hin, das in der lockeren Kopplung zwischen Wikipedia-Artikeln und klassisch bibliothekarischen Wissensobjekten durch Identifier wie ISBN oder PND vermutet wird.

Die Wikipedia ist in vielfacher Hinsicht modellhaft für die Generierung freier Inhalte im Internet. Gleichzeitig zeigen sich hier jedoch auch die Chancen und Risiken der Ausweitung bibliothekarischer Erwerbung auf derartige Inhalte.

11 Public Knowledge ist eine 2001 gegründete Non-profit Organisation mit Hauptsitz in Washington DC, die sich insbesondere für die Ausweitung urheberrechtlicher Schranken im Sinne der Informationsfreiheit und für das Prinzip des Fair Use (Begrenzung ausschließlicher Verwertungsrechte) einsetzt. http://www.publicknowledge.org/ (2.1.2012).

12 Vgl. Edgar u. Willinsky 2010.

13 http://de.wikipedia.org/wiki/Wikipedia (31.12.2011).

14 http://www.gnu.org/ (2.1.2012).

15 http://meta.wikimedia.org/wiki/Licensing_update/de (31.12.2011).

16 http://www.gbv.de/wikis/cls/SeeAlso (31.12.2011).

17 http://de.wikipedia.org/wiki/Hilfe:PND (31.12.2011).

18 http://commons.wikimedia.org/wiki/Commons:Deutsche_Fotothek/de (31.12.2011).

19 Heller 2010 
Open Access, Open Content und gemeinfreies Kulturerbe

Kommerzielle Plattformen wie Flickr ${ }^{20}$ und YouTube ${ }^{21}$ werden heute massenhaft dazu verwendet, nutzergenerierte Inhalte (in diesen Fällen Digitalfotos bzw. Videos) hochzuladen und anderen Nutzern zugänglich zu machen. Anders als im Fall der Wikipedia stehen diese Objekte jedoch nicht im Zusammenhang eines gemeinschaftlichen Werkes. Vielmehr pflegt jeder Nutzer ohne Verbindlichkeit gegenüber Dritten seine eigene Objektsammlung - ein wesentlicher Unterschied, den der häufig verwendete Oberbegriff des , user generated content' nicht benennt. Zudem handelt es sich - auch dies anders als im Fall Wikipedia bei den beiden genannten Plattformen um kommerzielle Unternehmen, die von ihren Nutzern das Einverständnis verlangen, ihre Objekte gewinnbringend zu nutzen, ohne ihnen im Gegenzug die langfristige Erreichbarkeit und Benutzbarkeit ihrer Objektsammlungen zu garantieren. Immerhin wird den Nutzern in vorbildlich einfacher Weise die Option eingeräumt, ihre Objekte unter einer selbstgewählten CreativeCommons-Lizenz zu veröffentlichen und somit eine pauschale Rechteeinräumung im Stil von Open Access bzw. freien kulturellen Werken vornehmen zu können. ${ }^{22}$

Die interne Suchfunktion der jeweiligen Plattformen, aber auch kommerzielle Websuchdienste greifen diese maschinenlesbare Lizenzierung auf, um gezieltes Recherchieren in einem Suchraum frei lizenzierter Objekte zu unterstützen. Auf der inhaltlichen Ebene fehlen vergleichbare Filter jedoch weitgehend. So lassen sich die auf den Plattformen durchaus anzutreffenden Open Educational Resources nur zu einem geringen Teil durch die Teilnahme am Programm http://www.youtube.com/edu identifizieren.

Aus den genannten Gründen lassen sich diese Plattformen nicht ebenso leicht an bibliothekarische Nachweisinstrumente anbinden wie die nichtkommerzielle, verhältnismäßig homogene Wikipedia. Dies wirft die Frage auf, wie Bibliothekare wissens- und erhaltenswerte digitale Objekte selektieren und erschließen könnten. Der enorme Wissenszuwachs, den solche einfach bedienbaren Plattformen beschleunigen, regt dazu an, diese Frage anders zu formulieren: Wie können kulturell oder wissenschaftlich engagierte Communities diese Selektion und Erschließung leisten, und wie können Bibliothekare im Zusammenspiel mit jenen Communities deren Erschließungsarbeit - die spontan in einem flüchtigen Webmedium wie dem Microblogging-Dienst Twitter aufgenommen wird $^{23}$ - begleiten, unterstützen und die Ergebnisse in den bibliothekarischen Entdeckungsraum einbringen? - Eine solche Participatory Librarianship $^{24}$ setzt neue Kompetenzen und ein neues Rollenverständnis für die bibliothekarische Erwerbungsund Erschließungstätigkeit voraus. Ohne diese Rollenentwicklung blieben die wissenschaftlich und kulturell werthaltigen, aber sehr heterogenen und dynamischen neuen digitalen Objektwelten des Netzes unverbunden neben den Entdeckungsräumen der modernen Bibliothek stehen. ${ }^{25}$

\subsection{Digitalisierungsprojekte und Gemeinfreies Kulturerbe}

Der Beginn der systematischen Retrodigitalisierung von Buchbeständen zur freien Zugänglichmachung via Internet kann mit Michael Harts „Projekt Gutenberg“ bereits auf das Jahr 1971 datiert werden.$^{26}$ Von Harts Freiwilligen-Initiative wurden bis heute zwar kaum mehr als 30000 Buchtitel digitalisiert, allerdings entwickelte es sich zum Kristallisationspunkt einer Reihe verwandter Initiativen wie Open Library, LibriVox, Wikisource, Zeno.org und Internet Archive, die gemeinfreien bzw. CC-lizenzierten Content im Netz erschließen und verbreiten. ${ }^{27}$ Die Nachnutzung von Content aus dem Projekt Gutenberg und ähnlichen Initiativen stößt aus deutscher Perspektive allerdings regelmäßig auf das Problem, dass die Grenze der Gemeinfreiheit im deutschen Urheberrecht anders definiert ist als in angelsächsischen Staaten.

Im deutschen und europäischen Kontext sind die Europeana sowie die Deutsche Digitale Bibliothek (DDB) zentrale Einstiegsportale, in denen retrodigitalisierte Kulturgüter aus Bibliotheken, Archiven, Mu-

20 http://flickr.com/ (31.12.2011).

21 http://youtube.com/ (31.12.2011).

22 http://www.flickr.com/creativecommons; http://www.youtube.com/t/creative_commons (31.12.2011).

23 Vgl. Microblogging und die Wissenschaft 2009.

24 Vgl. Participatory Networks 2007.

25 Ein Beispiel für dieses neuartige Rollenverständnis findet sich bei: Neumann u. Ulrich 2008

26 Hart 1992.

27 http://openlibrary.org; http://librivox.org; http://wikisource.org; http://www.zeno.org; http://www.archive.org (31.12.2012) 
seen und weiteren öffentlichen Einrichtungen archiviert und frei zugänglich gemacht werden. ${ }^{28}$ Diese neu gebündelten Informationsangebote beruhen auf einer Vielzahl von Digitalisierungsprojekten, die verschiedene Kultureinrichtungen, finanziert durch Eigenleistungen sowie Förderung durch öffentliche und private Geldgeber, seit Mitte der 1990er Jahre durchführten. ${ }^{29}$

Der US-Konzern Google startete mit der Google Buchsuche im Jahr 2004 ein Digitalisierungsprojekt, dessen finanzielle Ausstattung erstmals die Retrodigitalisierung großer Teile des gedruckten Kulturerbes anstrebt. ${ }^{30}$ Zwar formulieren auch ganze Nationen wie Frankreich und die Niederlande das Ziel der umfassenden Retrodigitalisierung ihrer Nationalliteratur innerhalb weniger Jahre, Google hat durch Kooperationen mit mehreren großen Universalbibliotheken (in Deutschland der Bayerischen Staatsbibliothek ${ }^{31}$ ) hingegen einen weltweiten Fokus. Die kooperierenden Bibliotheken erhalten jeweils Kopien für ihren eigenen Bestand. Seit 2010 werden den Bibliotheken weitgehende Rechte an diesen Digitalisate-Kopien eingeräumt, darunter die Indexierung durch Dritte zu kommerziellen Zwecken. ${ }^{32}$

Bei neueren Publikationen ermöglicht Google in Zusammenarbeit mit Verlagen den Rechteinhabern, ihre Werke via Google Buchsuche unter einer von ihnen selbst gewählten Lizenz zugänglich zu machen. ${ }^{33}$ Als gemeinfrei identifizierte Werke werden von Google als solche kenntlich gemacht und können als PDFDateien heruntergeladen, weiterverwendet und weiterverbreitet werden. Andere digitalisierte Werke können zwar im Volltext durchsucht, die gefundenen Ergebnisse jedoch nicht oder nur in Auszügen angezeigt werden.

Der Umgang der Google Buchsuche mit verwaisten Werken ist Gegen-stand wissenschaftlicher und juristischer Kontroversen. ${ }^{34}$ Verwaiste Werke sind noch nicht gemeinfreie Titel, deren aktuelle Rechteinhaber auch nach einer gewissenhaften Recherche (,reasonably diligent search ${ }^{435}$ ) nicht ermittelt werden können. In der Regel handelt es sich dabei um vergriffene Titel, die von einer öffentlichen Zugänglichmachung in digitalisierter Form in besonderem Maße profitierten. Google hat derartige Werke bereits in großem Umfang digitalisiert.

In Abgrenzung nicht zuletzt zu Googles Umgang mit verwaisten Werken haben das Internet Archive ${ }^{36}$, Yahoo und Microsoft zusammen mit zahlreichen Bibliotheken und Verbünden in den USA und Kanada die Open Content Alliance ins Leben gerufen. ${ }^{37}$ Sie stellt einen Pool von weit mehr als einer Million digitalisierter Buchtitel unter rechtlich unstrittigen Voraussetzungen weltweit zur freien Verfügung. Zum überwiegenden Teil handelt es sich dabei um gemeinfreie Werke.

Daneben existiert mit HathiTrust ein Netzwerk von US-Bibliotheken, die umfassende Digitalisierungsprogramme mit und ohne Beteiligung von Google betreiben. ${ }^{38}$ Sie stellen mehr als 10 Millionen Bände (Stand Januar 2012) in einem sowohl im Volltext als auch nach bibliographischen Kriterien durchsuchbaren Datenpool zur Verfügung, wobei die Definition der weltweit unstreitig anzubietenden Titel deutlich weiter gefasst wird als von der Google Buchsuche.

\subsection{Open Access}

Golden Road Journals, Green Road Repositories zur Selbstarchivierung durch Autoren sowie Open AccessMonographien werden von unterschiedlichen Diensten verzeichnet, aggregiert oder indexiert.

28 http://www.europeana.eu/portal; http://www.deutsche-digitale-bibliothek.de (31.12.2011).

29 Einen Überblick vermittelt die Seite http://www.kulturerbe-digital.de (31.12.2011).

30 Lewandowski 2005.

31 Vgl. Massendigitalisierung deutscher Drucke des 16. Jahrhunderts 2009.

32 Grimmelmann 2011.

$33 \mathrm{Ke} 2009$.

34 U. a. Kahle 2009 und IUWIS 2011.

35 Vgl. dazu die Resolution: Verwaiste Werke 2008.

36 http://www.archive.org/ (31.12.2011).

$37 \mathrm{http}: / /$ www.opencontentalliance.org/ (31.12.2011).

38 http://www.hathitrust.org/ (31.12.2011). 
Open Access, Open Content und gemeinfreies Kulturerbe

Das von der Universitätsbibliothek Lund betriebene Directory of Open Access Journals (DOAJ) ist ein weltweit anerkanntes Verzeichnis von Golden Road Journals. ${ }^{39}$ DOAJ betreibt einen OAI-Harvester ${ }^{40}$ und $^{2}$ kann daher auf seinen Seiten eine Suche in zahlreichen DOAJ-verzeichneten Journals auf Artikelebene anbieten und zudem im Hintergrund eine Langzeitarchivierung (in Kooperation mit der niederländischen Nationalbibliothek in Den Haag) gewährleisten. DOAJ legt bei der Aufnahme neuer Titel in das Verzeichnis scharfe Kriterien hinsichtlich Zugänglichkeit sowie Qualitätssicherung an. Das Verzeichnis ist spürbar anders zusammengesetzt als die Liste der als kostenlos zugänglich definierten Titel der Elektronischen Zeitschriftenbibliothek (EZB) der Universitätsbibliothek Regensburg. Zahlreiche Bibliotheksverbünde und Bibliotheken legen Titelaufnahmen neuer Zeitschriften systematisch anhand von DOAJ-Daten an, und auch Webangebote wie der JISC Inhaltsverzeichnis-Dienst TOCs ${ }^{41}$ speisen sich u. a. aus DOAJ-Daten. Seit 2006 bietet das Privatunternehmen Informatics (India) Ltd mit Open J-Gate eine Alternative zu DOAJ an, bei der es sich ebenfalls um eine Kombination aus Journal-Verzeichnis und -Harvester handelt. ${ }^{42}$

OpenDOAR ist ein von der Universität Nottingham sowie dem dortigen Centre for Research Communications betriebenes Verzeichnis von Open Access Repositories. ${ }^{43}$ Neben den ebenfalls in Nottingham betriebenen Diensten SHERPA RoMEO ${ }^{44}$ und JULIET ${ }^{45}$ gilt es als Rückgrat der weltweiten Infrastruktur der Open-Access-Selbstarchivierung durch Autoren (Green Road). OpenDOAR bietet eine Google Custom Search Engine zur Volltextsuche über alle verzeichneten Repositories an. Daneben existiert die Registry of Open Access Repositories (ROAR) an der Universität Southampton. ${ }^{46}$

Es kann mit Recht bezweifelt werden, ob DOAJ, OpenDOAR und die SHERPA-Projekte dauerhaft von wenigen Bibliothekaren an den jeweiligen Trägereinrichtungen betrieben werden können und sollten. Angesichts des weltweiten Informationswachstums und angesichts vorhandener, erprobter Werkzeuge zur kollaborativen Datenpflege erscheint uns ein Systemwechsel hin zu einem dezentralen Ansatz, bei kontinuierlicher Freigabe aller Daten unter einer Open Data-Lizenz, naheliegend.

Ein umfassendes weltweites Verzeichnis wissenschaftlicher Open Access-Monographien existiert zwar noch nicht, doch sind Verlags-Netzwerke wie Open Access Publishing in European Networks (OAPEN) bereits ein Hinweis darauf, wie Verlage ihren Content ins offene Web einbringen können. ${ }^{47}$ Das Netzwerk bietet ein Programm qualitätsgesicherter Open-Access-Buchtitel an, die sich auf der Website des Netzwerks durchbrowsen, von Bibliotheken als Katalogdatensätze fertig übernehmen sowie von OAIHarvestern und Webcrawlern indexieren lassen.

Harvester und Webcrawler indexieren übergreifend Open Access-Inhalte aus einer Vielzahl von Journals, Repositories und digitalen Bibliotheken. So wurde an der Universitätsbibliothek Michigan im Jahr 2002 der Harvester OAIster entwickelt, der 2009 von dem US-Dienstleister OCLC übernommen und als separate Datenquelle in den Portaldienst WorldCat integriert wurde. ${ }^{48}$ Parallel dazu entwickelte die Universitätsbibliothek Bielefeld die Bielefeld Academic Search Engine (BASE), die im Jahr 2004 in Betrieb ging. ${ }^{49}$ Bei BASE handelt sich inzwischen um eine Suchmaschine, die nicht nur Quellen indexiert, die sich nach dem OAI-Standard harvesten lassen.

Auch Dienste wie Thomson Reuters (ISI) Web of Science, Elsevier Scopus und Google Scholar indexieren in großem Umfang Open Access Journals. Google Scholar ermittelt zudem Open AccessParallelausgaben $\mathrm{zu}$ einem gegebenen Titel und hebt diese in einer geclusterten Ergebnisansicht hervor. Diese Dienste erlauben es allerdings nicht, gezielt nach Open Access-Materialien zu recherchieren. Darin

39 http://www.doaj.org/ (31.12.2011).

40 Zur Funktion eines solchen Harvesters vgl. Resource Harvesting within the OAI-PMH Framework 2004.

41 http://www.tictocs.ac.uk/ (31.12.2011).

42 http://www.openj-gate.com/ (31.12.2011).

43 http://www.opendoar.org/ (31.12.2011).

44 http://www.sherpa.ac.uk/romeo/ (1.2.2012).

45 http://www.sherpa.ac.uk/juliet/ (1.2.2012).

46 http://roar.eprints.org/ (31.12.2011).

47 http://project.oapen.org/ (31.12.2011).

48 http://www.oclc.org/oaister/ (31.12.2011).

49 http://base.ub.uni-bielefeld.de/ (31.12.2011). 
unterscheiden sie sich von föderierten Suchlösungen, bei denen dies von den lokalen Betreibern durch die Einbindung einschlägiger Suchindizes ermöglicht werden kann.

\section{Einbindung freier Ressourcen in Suchlösungen}

Im Bereich des Open Content überwiegt die dynamische Einbindung zusätzlicher Inhalte in die KatalogErgebnisanzeige (vgl. das Beispiel SeeAlso); im Bereich der Virtuellen Fachbibliotheken wird auch die Integration freier Inhalte in den Suchraum erprobt. Nichtkommerzielle Projekte wie die Wikipedia kooperieren direkt mit Bibliotheken. Zur Selektion von Content aus kommerziellen Plattformen, auf denen der Content nur teilweise frei zugänglich ist, besteht eine Möglichkeit in der Zusammenarbeit von Bibliotheken und informellen Communities auf der Suche nach neuen Wegen der Akquise und Erschließung großer Datenbestände.

Für das gemeinfreie Kulturgut stehen seit Beginn der systematischen Digitalisierungsaktivitäten umfassende Informationspools zur Verfügung, von denen einige in Standard-Austauschformaten für bibliothekarische Katalogdatensätze verfügbar sind (vgl. HathiTrust und $\mathrm{ZVDD}^{50}$ ). In Deutschland und Europa wird mit der DDB und Europeana begonnen, die Digitalisierung sowie die Erschließung und Zugänglichmachung der vorhandenen Daten und Projekte zu konsolidieren. Erschwerend wirken hier jedoch die international divergierenden urheberrechtlichen Rahmenbedingungen sowie die ungeklärten Fragen des Umgangs mit verwaisten und vergriffenen Werken.

Im Bereich Open Access beobachten wir die rasche Entwicklung einer internationalen Infrastruktur. Deren Eckpfeiler sind Institutional und Disciplinary Repositories sowie die zentralen Verzeichnisse von Golden Road Journals. Auf dieser Grundlage konnten auf Open Access spezialisierte Harvester und Suchdienste entwickelt werden. Zudem werden Open Access Inhalte von großen kommerziellen wissenschaftlichen Suchindizes berücksichtigt. In föderierten Suchlösungen können die lokalen Betreiber zudem die gezielte Selektierbarkeit von Open Access (und ggf. anderen frei verfügbaren Inhalten) gewährleisten.

Problematisch blieb bisher jedoch die Verfügbarkeit von Open Access in bibliothekarischen Rechercheinstrumenten. So finden sich im Verbundkatalog des $\mathrm{GBV}^{51}$, des größten deutschen Bibliotheksverbunds, zwar zahlreiche Open Access-Publikationen, doch werden diese im Regelfall nicht in den Lokalsystemen nachgewiesen. Zudem erfolgt keine sachliche Erschließung gemäß bibliothekarischer Standards. ${ }^{52}$ Die intellektuelle bibliothekarische Inhaltserschließung, auf der der traditionelle Online-Katalog beruht, stößt angesichts des Informationswachstums sowie der relativen Bedeutungszunahme von unselbstständiger und Grauer Literatur an Grenzen.

Neue Möglichkeiten bietet die Nutzung föderierter Suchlösungen. Durch Volltextindexierung, die sich über mehrere Indizes erstreckt, lassen sich Bibliothekskataloge ähnlich wie Websuchmaschinen benutzen. Metadaten - darunter auch intellektuell oder automatisch erzeugte Sacherschließungselemente - helfen dem Nutzer dabei, das Ergebnis seiner Stichwortsuche nachträglich einzugrenzen. Neben kommerziellen Lösungen wie OCLC WorldCat Local ${ }^{53}$, ExLibris Primo Central ${ }^{54}$, Serials Solutions Summon ${ }^{55}$ oder EBSCO Discovery Service (EDS) ${ }^{56}$ werden auch bibliotheksseitig, in Deutschland etwa vom GBV, kooperativ Suchindizes entwickelt und betrieben. Diese können von Bibliotheken in ihren lokal verwendeten Discovery-Lösungen nachgenutzt werden - unabhängig davon, ob es sich dabei um eine der oben genann-

50 http://www.zvdd.de/ (1.2.2012).

51 http://www.gbv.de/ (1.2.2012).

52 Vgl. hierzu ausführlich Hauke, Hötzeldt u. Rumler 2010.

53 http://www.oclc.org/worldcatlocal/ (2.1.2012).

$54 \mathrm{http}: / / \mathrm{www}$. exlibrisgroup.com/de/category/Primo_Central (2.1.2012).

$55 \mathrm{http}: / / \mathrm{www}$. serialssolutions.com/discovery/summon/ (2.1.2012).

56 Vgl. Kemner-Heek, Jansen u. Schweitzer 2010; http://ebscohost.com/discovery (2.1.2012). 
Open Access, Open Content und gemeinfreies Kulturerbe ten kommerziellen Lösungen oder um eine Eigenentwicklung zum Beispiel auf der Basis von VuFind ${ }^{57}$ handelt. $^{58}$

\section{$5 \quad$ Förderung von Open Access und Open Content durch Öffentliche Bibliotheken}

Der Aufbau digitaler Bibliotheken, die den Zugang zu einer Vielzahl elektronischer Ressourcen eröffnen, gehört seit einigen Jahren zum erweiterten Dienstleistungsangebot Öffentlicher Bibliotheken. Neben EBooks stehen dabei zunehmend auch Hörbücher und AV-Medien zur Ansicht bzw. zum Download bereit.

Die Einbindung frei zugänglicher Angebote im Sinne von Open Access und Open Content zählt bisher jedoch nur in Ausnahmefällen zum erweiterten Handlungsfeld Öffentlicher Bibliotheken. ${ }^{59}$ So wird auch in den IFLA / UNESCO Richtlinien „Dienstleistungen Öffentlicher Bibliotheken“" von 2005 neben den zentralen Kriterien „universell“, „allgemein“ und „spezifisch“ das Kriterium „,frei zugänglich“ nicht benannt, obwohl zugleich die „Rolle der Bibliothek als elektronisches Portal zur Informationswelt“" betont wird. ${ }^{60}$ Bisher bleibt Open Access auch aufgrund der definitorischen Eingrenzung auf wissenschaftliche Veröffentlichungen, die einem Peer-Review-Verfahren unterzogen wurden, weitgehend auf wissenschaftliche Informationseinrichtungen beschränkt. Im Bereich des Grünen Weges sind bisher von aktuell 127 institutionellen Repositories, die im Directory of Open Access Repositories (OpenDOAR) für den deutschen Bereich verzeichnet sind, keine Publikationsserver Öffentlicher Bibliotheken aufgeführt, auch wenn die OPUSDatenbank BIB des Berufsverbands Information Bibliothek e. V. eine Plattform für Publikationen zum öffentlichen Bibliothekswesen bietet. ${ }^{61}$ Diese thematische Fokussierung auf wissenschaftliche Einrichtungen spiegelt sich auch in den aktuellen Publikationen zu Open Access und Open Content als Teil des Bestandsmanagements wider. ${ }^{62}$

In den größeren Public Libraries des US-amerikanischen Bibliothekswesens, die aufgrund ihrer historischen Genese allerdings ein traditionell anderes Aufgabenspektrum abdecken, ist das Thema Zugang zu frei verfügbaren Online-Ressourcen hingegen bereits stärker in den Brennpunkt gerückt. Neben der Digitalisierung der eigenen historischen Bestände ist hier vor allem die Bereitstellung aktuellen Bildmaterials über Plattformen wie Flickr zu nennen. ${ }^{63}$ Der Nachweis von Informationsangeboten in den lokalen Systemen ist allerdings auch hier erst im Aufbau begriffen.

Welche Möglichkeiten bieten sich Öffentlichen Bibliotheken, die vorhandenen Content-Angebote für die lokale Nutzung gewinnbringend einzubinden und den freien und unbegrenzten Zugang zu Informationsressourcen im Internet zu erweitern?

Neben der oben beschriebenen Einbindung frei verfügbarer Ressourcen in die jeweiligen OnlineKataloge eröffnen sich für Öffentliche Bibliotheken vor allem drei Handlungsfelder: erstens die Bereitstellung von Inhalten im Open Access, zweitens die Beratung und Unterstützung lokaler Akteure beim elektronischen Publizieren sowie drittens die öffentliche Zugänglichmachung gemeinfreier Inhalte im Rahmen kooperativer Digitalisierungsaktivitäten. Zudem könnte die Recherche nach frei verfügbaren Informationsressourcen im Internet - etwa anhand recherchierbarer CC-Lizenzen - in die Veranstaltungen zur Vermittlung von Informationskompetenz eingebunden werden.

57 http://vufind.org/ (2.1.2012)

58 Kinstler 2010.

59 Neben Digitalisierungsaktivitäten von Stadtbibliotheken wie Mainz und Nürnberg sind hier insbesondere die Online-Angebote zu nennen, die das Ergebnis öffentlicher Kulturveranstaltungen darstellen. Vgl. etwa den Bereich „Open Content“ auf der Homepage der Stadtbücherei Stuttgart mit der stark genutzten Downloadmöglichkeit von Texten, Audiobooks und MedienBlogs. http://www5.stuttgart.de/stadtbuecherei/ (31.12.2011).

60 Dienstleistungen Öffentlicher Bibliotheken 2005: 47.

61 Vgl. http://www.opendoar.org/ (31.12.2011); http://www.opus-bayern.de/bib-info/ (31.12.2011).

62 Die Bedeutung von Open Access im Bestandsmanagement öffentlicher Bibliotheken wurde selbst in Publikationen, in denen der Aufbau digitaler Bibliotheken einen Schwerpunkt bildet, bisher nicht behandelt, ebenfalls nicht in der englischsprachigen Literatur, auch wenn dort der Fokus stärker auf elektronischen Medien liegt. Vgl. etwa Clayton u. Gorman 2006; Agee 2007.

63 Vgl. etwa die Angebote der New York Public Library auf der eigenen Homepage sowie auf der kommerziellen Plattform Flickr: http://digitalgallery.nypl.org/nypldigital/index.cfm u. http://www.flickr.com/photos/nypl/sets/ (31.12.2011). 
Die Förderung von Open Access als Teil des Bestandsmanagements ist dabei stark abhängig von dem jeweiligen historisch gewachsenen Bestands- und Aufgabenprofil der einzelnen Einrichtungen. So wird der Betrieb eigener Publikationsserver sich erst ab einem größeren Publikations-Output, der aus der jeweiligen Publikationstätigkeit und der Anzahl durchgeführter öffentlicher Veranstaltungen erwächst, empfehlenswert sein. Die Bereitstellung von Video- und Audioaufnahmen im Rahmen eines über die Homepage zugänglichen Text- und AV-Archivs ist hingegen für jede Einrichtung möglich.

$\mathrm{Zu}$ einem interessanten Aufgabenfeld könnte die technische und administrative Unterstützung lokaler Akteure (Vereine, Schulen, Verwaltung) im Prozess des elektronischen Publizierens werden. Sowohl die technischen Möglichkeiten als auch die lokale Nachfrage werden sich in diesem Bereich dynamisch weiterentwickeln, sodass hier für Öffentliche Bibliotheken je nach Versorgungslage die Bereitstellung und der Betrieb von Publikationstools empfehlenswert sein kann. Auch Informations- und Schulungsveranstaltungen für einen breiteren interessierten Adressatenkreis sind in diesem Zusammenhang denkbar.

Ferner sei auf mögliche Digitalisierungsaktivitäten insbesondere für größere Öffentliche Bibliotheken mit entsprechenden historischen Beständen hingewiesen, wie sie von Wissenschaftlichen Bibliotheken seit einigen Jahren im Regelbetrieb durchgeführt werden. Beispielhaft sind hier kooperative Digitalisierungsprojekte zu nennen, wie sie etwa von der Stadtbibliothek Nürnberg zusammen mit dem Germanischen Nationalmuseum durchgeführt wurden.$^{64}$ Insbesondere für die Lokal- und Regionalforschung sind hier wichtige Bestandsgruppen noch nicht erschlossen. Aufgrund des informations- und verwaltungstechnischen Aufwands wird sich allerdings vorrangig ein Outsourcing an externe Dienstleister bzw. eine Kooperation mit Einrichtungen empfehlen, die über die in diesem Bereich notwendige Erfahrung verfügen.

\section{$6 \quad$ Ausblick}

Eine stärkere Einbindung frei zugänglicher Informationsressourcen in die lokalen Online-Kataloge wird große Auswirkungen auf Erwerbung und Bestandsmanagement Öffentlicher Bibliotheken haben. Traditionell wichtige Themen wie Bestandsprofil, Richtzahlen für Bestandsgrößen und Approval Plans wären neu zu fassen und in Hinblick auf ein kundenorientiertes Informationsangebot fortwährend zu überprüfen.

Die größte Herausforderung und Chance besteht im Rollenwandel der Bibliothek zur Unterstützerin lokaler, aber auch virtueller Communities. Fachlektoren und Fachreferenten, Bestandsmanager oder ITSpezialisten werden dadurch nicht überflüssig, doch neben die klassisch bibliothekarischen Funktionen in der Verwertungskette der Literatur- und Unterhaltungsbranche tritt ein neuartiges partizipatives Bibliotheksengagement. Dieses Engagement kann von der Unterstützung lokaler Digitalisierungs-Aktivitäten über das Hosting freier Bildungs- und Informationsmaterialien reichen bis hin zur Anbindung von WissenschaftlerCommunities, die durch Wikis, (Micro)-Blogs oder Online-Literaturverwaltungsdienste eine vernetzte Auswahl- und Erschließungsarbeit leisten. Die Bibliotheken werden ihre jeweilige unterstützende, aktivierende oder ausstattende Rolle in der Interaktion mit diesen Communities finden müssen. Bereitschaft und Neugier zur Kommunikation mit den unterschiedlichen Akteuren sowie ein aktuelles Wissen über Technik und Spielregeln von Open Content, Open Access und gemeinfreiem Kulturerbe sind Voraussetzungen für das Gelingen dieses Zusammenspiels.

64 Vgl. http://www.nuernberger-hausbuecher.de/index.php?do=page\&mo=5 u. http://www.unitrier.de/index.php?id=14187\&no_cache=1\&tx_ttnews[tt_news]=9986\&tx_ttnews[backPid]=8895\&cHash=5f3c6b7a8b (31.12.2011). 


\section{Literatur- und Quellenverzeichnis}

Agee, Jim: Acquisitions go global. An introduction to library collection management in the $21^{\text {st }}$ century. Oxford: Chandos 2007.

Berliner Erklärung über den offenen Zugang zu wissenschaftlichem Wissen. 2003. http://oa.mpg.de/files/2010/04/Berliner_Erklaerung_dt_Version_07-2006.pdf (31.12.2011).

Brody, Tim u. Stevan Harnad: Comparing the Impact of Open Access (OA) vs. Non-OA Articles in the Same Journals. In: DLib Magazine 10 (2004) No. 6. http://www.dlib.org/dlib/june04/harnad/06harnad.html (31.12.2011).

Brody, Tim, Stevan Harnad u. Les Carr: Earlier Web Usage Statistics as Predictors of Later Citation Impact. In: Journal of the American Association for Information Science and Technology (JASIST) 57 (2005) 8. S. 1060-1072. http://EPrints.ecs.soton.ac.uk/10713/ (31.12.2011).

Clayton, Peter u. G. E. Gorman: Managing information resources in libraries. Collection management in theory and practice. London: Facet 2006.

Copy.Right.Now! Plädoyers für ein zukunftstaugliches Urheberrecht. In Zusammenarbeit mit iRights.info. Berlin: HeinrichBöll-Stiftung 2010. http://www.boell.de/downloads/2010-04-copy_right_now_zukunft_urheberecht.pdf (31.12.2011).

Dienstleistungen Öffentlicher Bibliotheken. IFLA/UNESCO Richtlinien für die Weiterentwicklung. Red.: Philip Gill. München: Saur 2005 (Bibliothekspraxis, 36).

Edgar, Brian D. u. John Willinsky: A Survey of the Scholarly Journals Using Open Journal Systems. In: Scholarly and Research Communication 1 (2010) 2. http://journals.sfu.ca/src/index.php/src/article/view/24/41 (31.12.2011).

Eysenbach, Gunther: Citation Advantage of Open Access Articles. In: Public Library of Science Biology 5 (2006) 4. http://www.plosbiology.org/article/info\%3Adoi\%2F10.1371\%2Fjournal.pbio.0040157 (31.12.2011).

Grimmelmann, James: GBS: No Exclusivity in the Scans? In: The Laboratorium, December 22, 2010. http://www.laboratorium.net/archive/2010/12/22/gbs_no_exclusivity_in_the_scans (31.12.2011).

Hart, Michael: The History and Philosophy of Project Gutenberg. Aug. 1992. http://www.gutenberg.org/wiki/Gutenberg:The_History_and_Philosophy_of_Project_Gutenberg_by_Michael_Hart (31.12.2011).

Hauke, Petra, Aline Hötzeldt u. Jana Rumler: Kataloganreicherung?! Vom Zugang zu Open Access-Publikationen über Bibliothekskataloge am Beispiel Hybrider Publikationen. In: Bibliothek. Forschung und Praxis (2010) 3. S. $293-305$. https://dochost.rz.hu-berlin.de/docviews/abstract.php?lang=ger\&id=37466 (31.12.2011).

Heller, Lambert: Sacherschließung von Literatur in und mit der Wikipedia - eine Spielidee. In: Biblionik [Weblog] 4.8.2010. http://biblionik.de/2010/08/04/sacherschliessung-mit-wikipedia-spielidee/ (31.12.2011).

IUWIS Redaktion: Judge Denny Chin weist das Google Book Settlement zurück. In: IUWIS [Weblog] 22.3.2011. http://www.iuwis.de/gbs_rejected (31.12.2011).

Kahle, Brewster: Google Claims to be the Lone Defender of Orphans: Not lone, not defender. In: Open Content Alliance (OCA) [Weblog] 7.10.2009. http://www.opencontentalliance.org/2009/10/07/google-claims-to-be-the-lone-defender-oforphans-not-lone-not-defender/ (31.12.2011).

Ke, Xian: Bringing the power of Creative Commons to Google Books. In: Inside Google Books [Weblog], 13.8.2009. http://booksearch.blogspot.com/2009/08/bringing-power-of-creative-commons-to.html (31.12.2011).

Kemner-Heek, Kirstin, Heiko Jansen u. Roswitha Schweitzer: Konkurrenzanalyse ausgewählter kommerzieller Suchindizes. Hrsg.: Hochschulbibliothekszentrum des Landes Nordrhein-Westfalen, Köln (hbz) und Verbundzentrale des Gemeinsamen Bibliotheksverbundes (VZG). Göttingen 2010. http://www.hbznrw.de/dokumentencenter/veroeffentlichungen/suchindizes.pdf (31.12.2011).

Kinstler, Till: Kooperativer Aufbau zentraler Lucene-Indices für lokale Portale. Präsentation, gehalten am 27.10.2010 auf dem Oracle Summit Bibliotheken 2010 in Weimar. http://www.gbv.de/Verbundzentrale/Publikationen/2010/2010/pdf/pdf_4483.pdf (31.12.2011).

Lewandowski, Dirk: Die Search Engines erobern die Buchwelt. In: Password 11 (2005). S. 29.

Massendigitalisierung deutscher Drucke des 16. Jahrhunderts. Ein Erfahrungsbericht der Bayerischen Staatsbibliothek. Von Markus Brantl, Klaus Ceynowa, Claudia Fabian, Gabriele Meßmer u. Irmhild Schäfer: In: Zeitschrift für Bibliothekswesen und Bibliographie 56 (2009) 6. S. 327-338.

Microblogging und die Wissenschaft. Das Beispiel Twitter. Steckbrief 4 im Rahmen des Projekts „Interactive Science“. Von Jana Herwig, Axel Kittenberger, Michael Nentwich u. Jan Schmirmund: Wien: Institut für Technikfolgen-Abschätzung der Österreichischen Akademie der Wissenschaften 2009 (ITA-Projektbericht, Nr. a524). http://epub.oeaw.ac.at/ita/itaprojektberichte/d2-2a52-4.pdf (31.12.2011).

Neumann, Vladimir u. Ivo Ulrich: Neue technische Entwicklungen auf dem Slavistik-Portal. http://www.slavistikportal.de/doku/ABDOS_Slavistik-Portal_2008.pdf (31.12.2011).

Participatory Networks: The Library as Conversation. Von R. David Lankes, J. L. Silverstein, S. Nicholson u. T. Marshall. In: Information Research 12 (2007) 4. http://informationr.net/ir/12-4/colis/colis05.html (31.12.2011). 
Resource Harvesting within the OAI-PMH Framework. Von Herbert Van De Sompel, Michael L. Nelson, Carl Lagoze u. Simeon Warner. In: D-Lib Magazine 10 (2004) No. 12. http://www.dlib.org/dlib/december04/vandesompel/12vandesompel.html (31.12.2011).

Steinhauer, Eric W.: Das Recht auf Sichtbarkeit. Überlegungen zu Open Access und Wissenschaftsfreiheit. Münster: MV Wissenschaft 2010. http://fiz1.fh-potsdam.de/volltext/aueintrag/10497.pdf (31.12.2011).

Verwaiste Werke. Verfahren für den digitalen Zugang zu einem bedeutenden Teil des kulturellen Erbes erforderlich. Bonn: Deutsche Unesco-Kommission 2008. http://www.unesco.de/resolution_verwaiste_werke.html (31.12.2011). 